\title{
La competitività dinamica degli ecosistemi territoriali. II caso degli eco-sistemi turistici multilocali tra Italia e Svizzera
}

\author{
LUCIANO PILOTTI ROBERTA APA** \\ ALESSANDRA TEDESCHI-TOSCHI ${ }^{* * *}$ IGOR SARMAN ${ }^{* * * *}$
}

\begin{abstract}
Obiettivo del paper: Questo paper si propone di analizzare in ottica "eco-dinamica territoriale" i driver emergenti della competitività dei sistemi d'impresa. Le risorse naturali, economiche e sociali proprie dei diversi sistemi territoriali locali e multilocali ed il loro sviluppo integrato assumono in effetti una crescente centralità negli attuali processi competitivi.

Metodologia: Considerando i precedenti studi sulla competitività territoriale e turistica, è proposto un modello strategico-ecologico elaborato attraverso lo strumento statistico della SEM. Tale modello che riprende la logica della "costellazione del valore" mette in luce come tutte le risorse e attività debbano interagire al fine di rendere competitivo un ecosistema multi-territoriale.

Risultati: Tale modello risulta efficace nel determinare i driver della competitività degli ecosistemi multilocali, facendo emergere anche una relazione tra le loro "performance competitive" ed eventuali Gap tra risorse possedute e attivabili.

Limiti della ricerca: Il modello si basa su valutazioni degli stakeholder e dunque potrebbe essere "viziato" da fattori soggettivi e tuttavia "compensato o attenuato" nei grandi numeri. Sarebbe inoltre opportuno ripetere la ricerca in diversi contesti e diversi periodi.

Implicazioni pratiche: Nel paper si propone un'applicazione empirica del modello all'Eco-Sistema del lago di Garda e a quello del Ticino, dal quale emergono indicazioni di marketing e di policy territoriali e multiterritoriali.

Originalità del lavoro: L'introduzione di un modello eco-dinamico per valutare l'efficiente utilizzo delle risorse territoriali, considerando oltre ai tradizionali fattori

* Ordinario di Economia e Gestione delle Imprese - Università degli Studi di Milano e-mail: luciano.pilotti@unimi.it

** Dottore di Ricerca in Business History and Management, Post Doc Researcher Università degli Studi di Milano

e-mail: roberta.apa@unimi.it

*** Professore a Contratto di Marketing dei prodotti digitali - Università degli Studi di Milano

e-mail: alessandra.tedeschitoschi@unimi.it

**** Dottorando di Ricerca di Marketing turistico- Università della Svizzera Italiana e-mail: igor.sarman@usi.ch
\end{abstract}


competitivi anche Internet ed in particolare le opportunità offerte per sviluppare relazioni tra imprese, sistemi e territori e tra questi e i consumatori.

Parole chiave: competitività turistica; ecosistemi turistici; driver della competitività; bundling e unbundling

Purpose of the paper: This paper aims to analyze in a "eco-territorial dynamism" emerging driver $s$ of business competitiveness. Natural economic and social resources, typical of the different local and multi-local regional systems and their integrated development are actually taking an increasingly central part in the current competitive processes.

Methodology: Considering the previous studies on territorial and tourism competitiveness we suggest a strategic model developed through the ecological-statistical tool of SEM. This model that resembles the logic of "value constellation" highlights how all the resources and activities must interact in order to make a competitive multi-regional ecosystem.

Results: This model is effective in determining the driver s of competitiveness multi-local ecosystems, revealing also a relationship between their "competitive performance" and any gap between the held and activatable resources.

Limits of the research: The model is based on assessments of stakeholders and thus could be "defective" by subjective factors, and yet it is "compensated or mitigated" in large numbers. It would also be appropriate to repeat the research in different contexts and different times.

Practical implications: This paper suggests an empirical model for the MultiLocale EcoSystem of Lake Garda and the Ticino, which brings out a territorial and multi-territorial marketing policy.

Originality of the study. The introduction of a dynamic economic model for evaluating the efficient use of territorial resources, considering besides the traditional competitive factors also the Internet and in particular the opportunities to develop relationships between businesses, and territories and between these systems and consumers.

Key words: competitiveness of tourism; ecosystems tourism; driver of competitiveness; bundling and unbundling

\section{Competitività e innovazione nel turismo: alcune ipotesi e scenari emergenti}

È ormai evidente che la complessità, intesa come varietà (nello spazio), variabilità (nel tempo) e «indeterminazione» (nelle leggi causali), sia il tratto distintivo degli attuali contesti competitivi, che richiedono sempre più eccellenza e integrazione dei servizi per migliorare attrattività e competitività di contesti ecologico-territoriali, favorendo il rinnovamento e lo scambio di risorse attraverso la triangolazione tra talenti, tecnologia e territorio à la Florida. È altrettanto noto che in tali contesti la competitività delle imprese, così come quella dei network e dei sistemi, si basi sempre più sulla capacità d'individuare e soddisfare i segmenti di 
mercato emergenti, quindi guardando ai fattori potenziali più che a quelli derivabili linearmente dal passato.

Tali politiche, peraltro, stimolano un continuo aumento della stessa complessità, dilatando la varietà (differenziazione dei mercati e diversificazione dell'offerta), la variabilità (contrazione dei cicli di vita dei prodotti) e la flessibilità di risposta ad una domanda sempre meno standard e prevedibile. La sostenibilità nel medio termine di questi vantaggi competitivi richiede quindi ai diversi player di attuare politiche di posizionamento e ri-posizionamento continuo che rendano la propria offerta unica ed inimitabile tanto nel mondo dei servizi, quanto in quello dei prodotti materiali. Ciò rende necessarie adeguate strategie di creative marketing, finalizzate alla disarticolazione (unbundling) e riconfigurazione (bundling) delle diverse componenti d'offerta (d'impresa, di network, di sistema e locale) per rispondere meglio dei concorrenti alle aspettative dei diversi segmenti di utenti finali anche e soprattutto attraverso una più sostenibile e diffusa interazione con questi.

Tali aspetti emergono con particolare intensità nel settore del turismo nel quale le risorse naturali (endowed) e quelle create del territorio, quale «destinazione turistica», non sono più di per sè sufficienti per affrontare le nuove dinamiche competitive, qualora non «attivate» e gestite in modo creativo, dai singoli operatori, dal sistema e dallo stesso utente-consumatore anche e attraverso adeguate politiche di destination management e di social community marketing.

Il settore del turismo da alcuni anni è infatti interessato da significativi cambiamenti che rendono il binomio innovazione-territorio un driver critico della competitività dei sistemi d'offerta. Ci riferiamo:

- ai cambiamenti dei modelli di consumo caratterizzati dalla crescente domanda di varietà, qualità, contenuti esperienziali, emozionali e di sense making. Contenuti di per sé unici e non standardizzabili, dando valore a significati, a legami e a identità auto-generate. L'emergere di questa domanda, che qui definiamo come post moderna, si accompagna ad una progressiva articolazione delle forme d'offerta, introducendo, in alcuni casi, una virtuosa circolarità tra creatività domandata e creatività offerta;

- l'apertura al turismo di molti mercati, sino ad oggi non accessibili in senso commerciale, economico e geografico e al conseguente aumento delle alternative/forme d'offerta;

- alla progressiva disintermediazione dei tradizionali canali distributivi che si accompagna alla diffusione della multicanalità dell'offerta e della co-produzione da parte della domanda. Alla base di questa opportunità di sviluppo c'è la capacità degli operatori di "ascoltare e/o conversare" con i consumatori, le cui scelte sono sempre più community based, volatili e poligamiche. Ciò richiede che la progettazione e la governance dell'offerta turistica si basi sempre più sulla «valorizzazione» delle componenti relazionali, rendendo l'utilizzo del Web e di tutte le sue potenzialità (dalla profilazione della domanda all'immaginazione, produzione e promozione collaborativa dell'intera esperienza turistica) una risorsa competitiva di grande importanza. 
Le dinamiche competitive, cui si è accennato, hanno progressivamente aumentato la rilevanza dei sistemi territoriali locali, data l'interdipendenza (logistica, informativa e di marketing, oltre che di conoscenza e fiducia) tra sistemi, istituzioni e singoli attori e dalla molteplicità di relazioni tra componenti materiali ed immateriali dei sistemi d'offerta. Relazioni che nel complesso hanno modificato le traiettorie evolutive del settore turistico, fino a ridisegnare gli ambiti competitivi d'integrazione tra più dimensioni micro, meso e macro. In tale settore, infatti, la competizione si svolge non solo o non tanto tra singoli player e/o località, ma sempre più tra eco-sistemi (territoriali, multi territoriali e/o regionali), la cui competitività si fonda non solo sulle tradizionali caratteristiche ambientali ed attributi identitari e d'immagine forti, oltre che su vantaggi «dinamici e costruiti», ma anche e soprattutto sulle capacità d'imprese, sistemi e territori di rispondere in modo differenziato alla domanda di creatività proveniente dai mercati.

Ad una prima sintesi dunque avremo che le strategie emergenti e necessarie per competere nei mercati turistici post moderni vanno interpretate alla luce di un concetto di destinazione e di servizio turistico più ampio di quello tradizionale. Il primo fa riferimento ai sistemi turistici multi-locali, che superano le tradizionali aggregazioni geo-politiche in funzione dei diversi target di domanda; il secondo al bene turistico quale bene complesso, composto da beni appropriabili e non appropriabili (commons). In tal senso, potremmo dire, non esiste una one best way per il turismo, ma esistono diversi modelli creativi dai quali possono derivare più combinazioni di servizi e di prezzo-qualità (e innovazioni) economicamente sostenibili e conseguentemente più possibilità di diversificazione delle forme d'offerta, ottenute disaggregandone e ri-aggregandone le diverse componenti dentro ed attraverso nuovi sistemi multi-territoriali e multi servizio. Ciò rende necessario il passaggio da una logica competitiva individuale o puntuale (territoriale) ad una di sistema multi-locale e richiede alle imprese la capacità di agire trasversalmente tra sistemi micro, vale a dire la singola impresa o rete, e sistemi macro, quali i sistemi territoriali multilocali, utilizzando nuovi strumenti analitici, strategici ed operativi che in particolare valorizzino la qualità e il posizionamento delle singole componenti, ma anche (e soprattutto) di quella complessiva di «sistema ecologico multilocale» ${ }^{1}$. Quest'ultima, in particolare, deriva dall'equilibrio dinamico tra diversi livelli di sistema (territori, reti, imprese ed altri stakeholder) che può produrre esternalità a volte negative (es. congestione dei servizi), ma spesso anche positive $^{2}$, come nel caso delle infrastrutture e della comunicazione, solo per fare due

1 Ecosistema è un sistema in cui i singoli soggetti (individui, istituzioni, comunità) si muovono con azioni strategiche sia passive che attive e pongono differenti basi alla generazione di valore per la governance dell'interdipendenza. Un sistema in cui la creazione del valore avviene in un mondo non-zero-sum-based dominato da innovazione e crescita comune. In proposito si veda Pilotti (2001, 2006). Sugli eco-sistemi turistici si veda, Pilotti et al., $(2010,2011)$

2 La qualità competitiva che emerge dagli eco-sistemi turistici multilocali (ESTmL) pertanto non è una semplice somma di «singole qualità», ma va intesa come «qualità 
semplici esempi. Esternalità positive che si esprimono attraverso interdipendenze tali da produrre la condivisione di valore derivante da storia, tradizioni o consuetudini ed economie locali che sono virtuose condivise e comuni, e che richiedono adeguati sistemi di Governance (dal basso) e di Government (dall'alto) interni ed esterni nella valorizzazione di tali eco-sistemi turistici complessi, attraverso lo sviluppo delle loro capacità creative, individuali, collettive e organizzative. In tal modo i diversi stakeholder saranno in grado di ricercare e sperimentare continuamente la propria varietà e dunque costruire una propria originalità da rendere complementare con altre e scambiarla per «governare» la continua domanda di innovazione. In tale processo di scambio, alcune componenti dei sistemi d'offerta si svilupperanno e altre deperiranno, ma qui risiede il rischio e l'opportunità del matching tra le diverse componenti dei sistemi d'offerta turistici per rispondere alla crescente domanda di varietà. Qui risiede l'enorme potenziale competitivo degli eco-sistemi-multilocali e la sua sostenibilità rispetto alle risorse endowed e created. Il tutto nell'ottica della soddisfazione e della creazione di fiducia dei e tra i diversi stakeholder e tra diversi territori per accrescerne l'attrattività e la valorizzazione e di conseguenza il riacquisto da parte di consumatori-utenti, sempre più evoluti, discontinui ed iper-segmentati, che si confrontano con un'offerta sempre più ampia, flessibile ed accessibile per decomponibilità e ricomponibilità del servizio. Da qui la necessaria revisione ed integrazione dei modelli di valutazione della competitività turistico-territoriale in senso ecologico.

Il lavoro affronta questi temi partendo da una sintetica review della letteratura sui driver della competitività (par.1), mettendone in luce la necessità di una loro integrazione per tenere conto degli attuali cambiamenti nella competizione globale che, di fatto, modificano gli stessi driver della competitività. Va osservato che il concetto stesso di competitività assorbe crescente complessità essendo " $a$ complex concept because a whole range of factor account for it. Competitiveness is both a relative concept (i.e compared to what?) and is multi-dimensional (i.e, what are the salient attributes or qualities of competitiveness?)" (Dwyer e Kim, 2003) e che non esiste in letteratura un'univoca definizione di competitività turistica. Tuttavia ricomponendo i vari contributi è possibile affermare che una destinazione è competitiva se dispone di un appeal capace di generare un'esperienza turistica superiore a quella offerta da territori alternativi e quindi concorrenti. Questa superiorità viene rilevata, oltre che dai tradizionali indicatori di performance (indice di flussi in entrata, indice di permanenza media, tasso di occupazione lorda, reddito prodotto, pil turistico, ecc.), anche dalla crescente integrazione sinergica tra le risorse attrattive primarie (naturali, umane, artificiali) della destinazione, le infrastrutture che ne agevolano la fruizione, le imprese turistiche, le industrie complementari e di supporto a quella turistica, le istituzioni, la popolazione residente e la domanda turistica.

aggregata o aggiunta» superiore alla loro sommatoria lineare, il cui livello deriva dalla coerenza tra qualità di sistema e qualità dei singoli servizi. 
In tale direzione, il lavoro propone quindi un modello integrato per l'analisi della competitività (par. 2), alla luce di un concetto di destinazione turistica più ampio, che come si è accennato fa riferimento a sistemi turistici multi-locali. Il modello riprende la logica della "costellazione del valore" delle destinazioni turistiche facendola evolvere in senso ecologico. In particolare, questo modello, confrontando la dotazione di diverse categorie di risorse (naturali, create, di supporto, d'immagine, economiche e tecnologiche) dei sistemi territoriali consente di valutarne le capacità attrattive eco-sistemiche attuali e potenziali e dunque la loro competitività. Il modello, composto da 47 variabili, è stato validato attraverso uno Structural Equation Modeling System (par. 3) con riferimento ai sistemi turistici multi territoriali del Lago di Garda e dei Laghi del Ticino che fanno parte della più ampia Macro-Regione-Europea dei laghi del Nord.

\section{La competitività dei sistemi turistici tra territorio, risorse endowed e created e destination management}

La centralità del territorio e dei sistemi territoriali nella competizione globale emerge chiaramente in letteratura. Alcuni autori si sono focalizzati su particolari driver competitivi, altri hanno proposto modelli più completi, ma pur sempre riferiti alle «destinazioni turistiche», traslando concetti dal macro al micro, non considerando in tal modo le esternalità derivanti dalle inter-connessioni macro=> meso $=>$ micro $=>$ macro $(. .$.$) che fanno dell'ecologia sistemica e della creatività uno$ dei driver principali della competitività dei sistemi turistici multilocali nei mercati globali.

In particolare, per quanto concerne i driver della competitività delle destinazioni turistiche, questi sono stati di volta in volta individuati in fattori strutturali ed ambientali (Govers e Go, 1999), in aspetti "soft" quali la qualità del capitale sociale, le relazioni e la capacità innovativa (Senn 2002; Buhalis, 2000), nelle policy territoriali (Poon, 1993), nelle politiche dei singoli operatori e negli stessi comportamenti della domanda (Rispoli e Tamma, 1995; Valdani e Guenzi, 1998). Particolare attenzione è peraltro dedicata all'immagine del territorio quale codeterminante della qualità percepita (Grönroos, 1994) che risulta dall'interazione tra diverse componenti materiali ed immateriali (prodotti, eventi, utenti, contesti, atmosfere e così via). Componenti non necessariamente collegate tra loro o congruenti nel significato, che richiedono adeguate politiche finalizzate a creare un'immagine coerente e condivisa, quale risorsa di fiducia dalla quale dipende l'attrattività ed il riacquisto di un prodotto/località turistica (Ostilio, 2000). La coerenza tra immagine ed identità (industriale e/o turistica) dei sistemi territoriali (patrimonio «interno» di risorse e competenze dei sistemi stessi) è considerata un ulteriore driver competitivo (Kotler e Gertner, 2002), così come il brand collettivo, quale risorsa relazionale che crea e mantiene un vantaggio sostenibile nel tempo (Ambler, 1995, Fournier, 1998). 
Quanto agli schemi analitici più complessi che considerano diversi driver di competitività, questi generalmente sono riconducibili ad approcci «neostrutturalisti», quale quello di Crouch e Ritchie (1999; 2003), o ad approcci «resource based» (Dwyer e Kim, 2003). In particolare, Crouch e Ritchie considerano quali driver competitivi le core resources, $i$ supporting factors, le policy territoriali e il destination management, riconducendo la competitività di una destinazione turistica non soltanto dalla sua dotazione di risorse naturali, storiche e culturali, ma anche dalle competenze e sinergie locali che la stessa è in grado di atttivare. Dwyer e Kim propongono invece un framework (Pilotti et al., 2011) in cui i comportamenti dei consumatori e la struttura della domanda rappresentano le determinanti principali della competitività delle destinazioni turistiche, oltre alle risorse naturali, artificiali e di supporto. Tali modelli tuttavia esaminano i driver della competitività solo tramite un confronto tra i valori medi delle valutazioni che ogni singolo item ha ricevuto dai principali stakeholder dell'area in oggetto, non esplorando le relazioni tra di essi, come nel modello da noi proposto.

Più di recente Caroli (2009) ha ricondotto la competitività di una destinazione turistica alla sua meta-capacità di «creare valore» per la domanda, mettendo il turista nelle condizioni di co-produrre la propria esperienza, differenziandola in tal modo dalle offerte concorrenti. In tale ottica, la qualità competitiva di una destinazione turistica è determinata in base ad un preciso mix di capacità, identificate in base alle attività fondamentali che il turista svolge durante la propria esperienza turistica, organizzate «à la Porter» in una «catena del valore delle capacità». Il modello riprendendo alcuni concetti di economia territoriale e delle città in una prospettiva ecologica sviluppati da Pilotti (2001; Pilotti e Ganzaroli, 2009), distingue le capacità primarie dalle capacità trasversali. Le prime sono le generatrici fondamentali del valore in quanto influenzano direttamente il modo in cui il turista fruisce dell'offerta di una località (capacità di comunicare, informare, accogliere, ospitare, intrattenere e stupire, etc.). Le capacità trasversali comprendono sia la capacità di integrare le componenti più rilevanti dell'offerta di una destinazione turistica (integrazione interna) e queste ultime con quelle di altre destinazioni vicine (integrazione esterna), sia la capacità di pianificare e sostenere uno sviluppo turistico sostenibile e le caratteristiche socio-economiche della destinazione turistica.

Nel complesso dunque la competitività emerge in tutte le sue dimensioni nell'ambito dei sistemi turistici territoriali, la cui centralità nella competizione globale è ampiamente riconosciuta. Si tratta, in effetti, di sistemi multi settoriali dove la domanda manifesta da un lato una crescente richiesta di prodotti caratterizzati da contenuti di differenziazione identitaria (ambientali, sociali, storici e culturali, etc.) e dall'altra una sempre minore intermediazione tra player settoriali (alberghi, tour operator, etc.) e territorio (vacanza come opportunità di apprendimento), facilitando i processi di co-creazione dell'esperienza d'acquisto e di consumo. Tutto ciò richiede una governance efficace dei sistemi turistici territoriali (STL), delle loro risorse endowed \& created, in un processo che è sempre più di tipo bottom up che consente di condividere con i diversi stakeholder gli obiettivi di sviluppo, mantenimento e sostenibilità della destinazione turistica prescelta. Ł̀ 
inoltre riconosciuto che sono le destinazioni che competono e non più le singole imprese e che la capacità di attrarre risorse (flussi turistici, nuovi capitali e nuove competenze), non deriva più di per sé dall'azione di singole componenti territoriali (imprese turistiche o istituzioni di singole aree), ma è il risultato di una loro attività sistemica, integrata in un coerente piano di sviluppo turistico, che esprime e compone i diversi interessi espressi dal territorio spesso entro logiche di autoorganizzazione e vocazionali e dunque favorendo ecologie emergenti (Govers e Go, 1999; Weaver e Oppermann, 2000; Flagestad e Hope, 2001).

Non emerge tuttavia la necessità di «disaggregare» i diversi mercati turistici e «ricomporli» in modo flessibile in eco-sistemi territoriali finalizzati a soddisfare la domanda in modo sostenibile. La nostra idea è che:

a. la competizione a livello internazionale non sia, infatti, limitata, nè possa limitarsi, alle tradizionali aree geo-politiche o geo-territoriali, ma si svolga tra più territori meta-organizzati, al cui interno la creazione del valore avviene in un mondo non-zero-sum-based dominato da innovazione e crescita comune (Pilotti, 2006), i cosiddetti «Eco-Sistemi Turistici Multi-Locali».

b. la competitività degli Eco-Sistemi turistici multilocali si fondi non solo sulla ricchezza e sul dinamismo di fattori inimitabili dei quali il «singolo» territorio diviene contenitore «ecologico» (Pilotti, 2006) - che affonda innanzitutto nella storia e nelle istituzioni diffuse, nella capacità di alimentare con le proprie radici nuovi modi di fare, produrre e consumare - ma anche su nuove modalità di connessione tra gli individui e le loro istituzioni e tra questi ultimi e quelli di altri territori contigui.

Il passaggio da una logica competitiva individuale o puntuale (territoriale) ad una di Eco-Sistema multi-locale, presuppone l'esistenza di obiettivi comuni verso i quali far convergere capacità comunicative e progettuali, risorse umane e finanziarie ed energie morali.

È necessaria inoltre, la continua ricerca di un adeguato livello di consenso (mobilitazione, consultazione, concertazione e legittimazione degli attori) nonché la capacità degli attori coinvolti di definire e rispettare regole chiare di comportamento ed azione verso una superiore social choesion. Si tratta ad evidenza di un concetto di offerta turistica più ampio e profondo, in base al quale si allargano notevolmente i confini dell'analisi delle fonti di competitività e degli interventi per la loro implementazione per una sorta di multidimensionalità dinamica dell'oggetto di indagine. Da qui nasce anche la necessità di adottare nuovi metodi per misurare la competitività delle destinazioni turistiche, integrando i modelli qualitativi più sopra brevemente ricordati. 


\section{Un modello per valutare la competitività dinamica multidimensionale degli EcoSistemi Turistici Multi Locali}

La relazione tra competitività e risorse endowed e created emerge compiutamente adottando la prospettiva ecologica nell'analisi della competitività dei sistemi turistici. Tale approccio, tra l'altro si basa sull'idea che la competitività territoriale non possa emergere dal confronto statico delle prestazioni dei diversi territori e neanche come semplice somma della competitività delle imprese locali per cui non è sufficiente adattare approcci «macro» a contesti locali, o approcci «micro» a contesti più ampi (territori).

È invece necessario adottare un approccio che preveda un livello analitico intermedio tra il «micro» (imprese) ed il «macro» (nazioni), un meso-livello economico in cui sono coinvolti anche aspetti sociali e relazionali oltre che le connessioni tra mercati e non-mercati capaci di sollecitare la formazione autoorganizzata sia delle vocazioni, sia l'attivazione di nuove competenze. In tal senso, il territorio diventa la vera risorsa per lo sviluppo.

Il suo valore non è statico, ma dinamico e pertanto fattore di creatività e di innovazione potenziale, mobilitabile attraverso interventi e progetti coordinati, generando scambi sia al proprio interno, sia con aree geografiche esterne, al fine di creare valore per la comunità di riferimento ${ }^{3}$.

I territori assumono in tal modo, il ruolo di tessuti connettivi e spazi relazionali, ovvero contesti caratterizzati da dense interazioni, preziose per la produzione di valore economico, sociale e relazionale (Paiola, 2006) alimentato da diffusi inspessimenti comunitari. Tali aspetti vanno ad evidenza considerati nell'analisi della competitività di una destinazione turistica.

In quest'ottica nelle pagine che seguono proponiamo un modello per la definizione dei driver che maggiormente rendono più competitivo un ESTmL. Tale modello riprende la logica della "costellazione del valore", superando in tal modo la tradizionale "catena del valore" delle destinazioni turistiche (Fig. 1). Si tratta, infatti, di un modello strategico-ecologico dal quale emerge come tutte le risorse e attività debbano interagire al fine di rendere competitivo ed attrattivo un ecosistema multiterritoriale, multi-strato e multi-periodale.

Tale modello valuta, in ottica eco-dinamica, l'efficiente utilizzo delle risorse territoriali endowed e created e considera oltre ai tradizionali fattori competitivi anche Internet ed in particolare le opportunità offerte per sviluppare relazioni tra imprese, sistemi e territori e tra questi e i consumatori, direttamente o attraverso i social media.

3 Tale affermazione prende le mosse da contributi che si focalizzano sul ruolo della variabile "relazionale" nella governance territoriale [Caroli, 1999; Van den Berg e Braum, 1999;] e che, in particolare, riconducono il successo competitivo dei territori alla capacità di gestire lo "spazio globale dei flussi", piuttosto che nella sola valorizzazione delle risorse endogene in chiave atomistica.. 
Fig. 1: Modello di Ecologia del valore

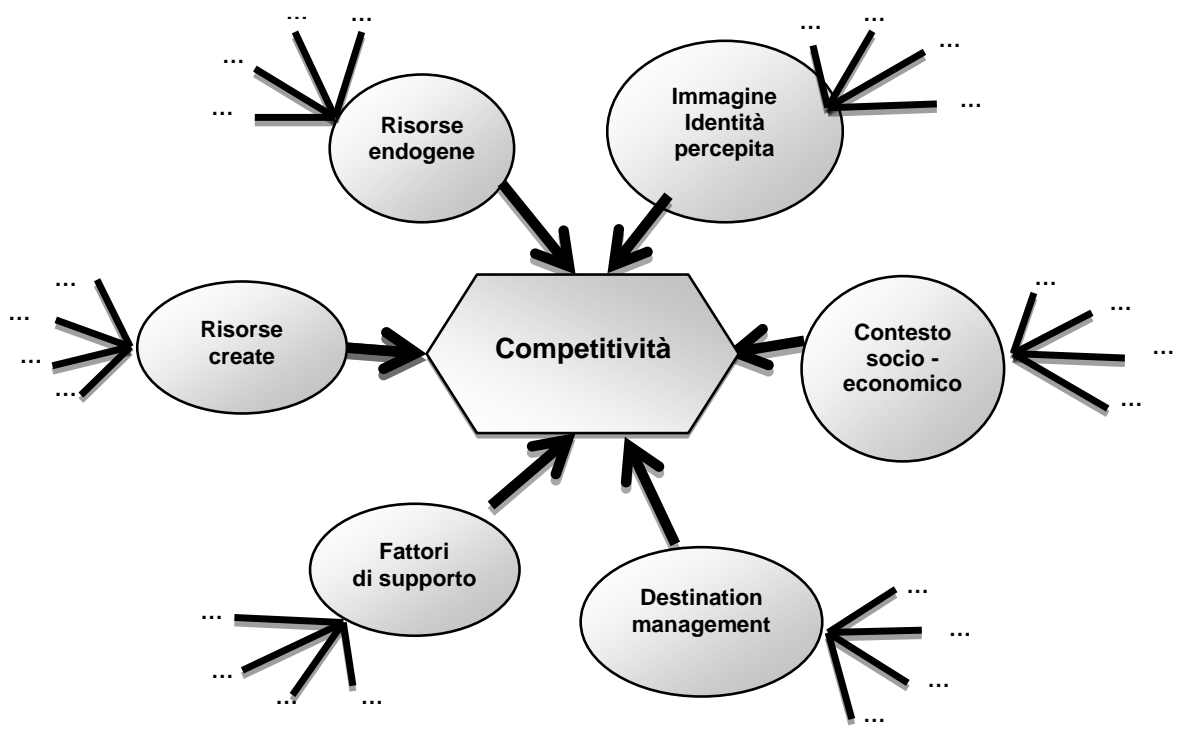

Fonte: ns. elaborazione

Il modello è composto da 7 macro variabili suddivise seguendo l'impostazione del modello integrato per la valutazione della competitività delle destinazioni di Dwyer e Kim (2003), introducendo la macro variabile Web e ICT, che a nostro avviso completa il set dei driver che rendono competitivo un sistema turistico. Si tratta in particolare delle:

- Risorse endogene: quali il patrimonio naturale e culturale. Spesso costituiscono la vera fonte del vantaggio competitivo di un'area essendo scarsamente imitabili e quindi altamente differenzianti Esse sono costituite dal patrimonio naturale (clima, flora e fauna, ambiente naturale etc.) e culturale (storia, costumi, monumenti, cucina, tradizioni, arte, musica, etc. $)^{4}$.

- Risorse create: si tratta delle strutture turistiche, di per sè imitabili, riproducibili e rinnovabili. Tali risorse, essendo create, possono cambiare nel tempo e, rispetto alle risorse endogene sono più rinnovabili, anche se in certi casi esse possono essere legate a particolari significati storici o artistici (particolari opere archeologiche o d'arte) e quindi non essere rinnovabili.

- Fattori di supporto: determinano la capacità produttiva del settore e la carrying capacity del sistema quali le infrastrutture generiche, la qualità dei servizi,

4 Il patrimonio cultura rappresenta una potente forza attrattiva nella prospettiva del visitatore (Murphy et al., 2000). 
l'accessibilità delle destinazioni, l'ospitalità e i legami con i mercati (Dwyer e Kim, 2003). Le organizzazioni pubbliche e private che possiedono specifiche capacità non facilmente imitabili, possono essere un'importante fonte per sostenere i vantaggi competitivi (Barney, 1991; Prahalad e Hamel, 1990), in quanto provider di infrastrutture che supportino le attività turistiche.

- Destination management: include tutti i fattori che regolano ed influenzano lo sviluppo di una destinazione turistica (Dwyer e Kim, 2003), gestendo nel lungo periodo il matching tra gli interessi dei diversi stakeholder (Buhalis, 2000).

- Contesto socio-economico: rappresenta un fattore critico di successo per un sistema turistico, come dimostrato anche da vari studi a livello regionale che sono basati sulla nozione per cui il processo innovativo delle imprese non può essere separato dall'ambiente in cui sono inserite. Tale fattore include tra l'altro il sistema normativo, le relazioni tra gli attori pubblici e privati all'interno della regione.

- Web e le ICT: intesi come facilitatori dello sviluppo della domanda e dei processi di co-creazione e condivisone dell'intero processo di acquisto-consumo e post consumo dei servizi turistici.

- Immagine e identità percepita: il brand territoriale rappresenta senza dubbio uno strumento indispensabile attraverso cui capitalizzare il valore comunicativo e relazionale di un ESTmL, in quanto ne differenzia e ne garantisce la qualità dei prodotti/servizi, contribuendo in tal modo alla formazione delle preferenze e al consolidamento della qualità percepita (Valdani e Ancarani, 2000).

La rilevanza e le connessioni tra queste variabili, come vedremo, sono state analizzate tramite una factor analysis, che come è noto, consente di ottenere una riduzione della complessità del numero dei fattori che spiegano l'attrattività di un sistema turistico, spiegando i legami, le interazioni e le dipendenza tra le variabili osservate. La nostra idea, infatti, è che la connessione e l'interazione che configurano le interdipendenze di tutti i fattori evidenziati consenta di co-creare (ad attori, istituzioni e meta-organizzatori) - anche per processi auto-organizzati - qualità e valore per l'ESTmL, determinandone l'attrattività e la competitività. Il valore e la qualità dell'intero sistema saranno maggiori quanto più sono equilibrate le sue componenti sia in relazione all'apporto diretto al valore (fattori di appropriabilità), sia in relazione alla riduzione dei costi complessivi (fattori di esternalità) e all'innovazione (apporto di knowledge, per esempio attraverso valorizzazione identitaria) (Pilotti, 2001).

Il soddisfacimento dei diversi stakeholder crea inoltre fiducia ed incentivi ad investire nel territorio per aumentare l'attrattività e rigenerare le esternalità, che saranno in parte internalizzate dagli attori dinamici e dagli innovatori. Il potere differenziante e il potenziale competitivo dipendono sempre più da elementi non materiali ed intangibili, in particolare dall'identità, dalle conoscenze trasferite e dalla fiducia, spesso incorporati in artefatti e nei servizi erogati dall'area turistica locale. Tale approccio consente di tener conto dell'esigenza sistematica di cambiamento attualmente richiesta dalla competizione internazionale per rispondere alla crescente domanda di varietà e qualità e dunque all'allungamento delle code di offerta e di 
domanda. Il primo aspetto, infatti, si riferisce in particolare all'emergere anche nel settore turistico del fenomeno della così detta "coda lunga", il secondo fa riferimento alla domanda di prodotti multitasking (che rispondano ad aspettative diverse e variabili nel tempo e nello spazio). Questi due aspetti saranno approfonditi nelle pagine che seguono, con riferimento al caso dell'ESTmL del Garda e a quello del Ticino. In tale ottica un ESTmL è tanto più competitivo quanto più riesce a rispondere ad una domanda sempre più segmentata e pur tuttavia interconnessa da specifici nodi che vanno via via identificati e resi attivi, modificando le forme di erogazione dei servizi. Ciò richiede, come già detto, un matching tra tutte le componenti del sistema, per valorizzare l'offerta complessiva, differenziando ed amplificando le identità delle singole componenti come coerente up-grade dell'identità originaria.

\section{Metodologia, definizione del modello e risultati}

\section{1 Contesti di riferimento}

Il modello è stato testato e validato con riferimento l'ESTmL del Lago di Garda e quello dei Laghi del Ticino che si caratterizzano per potenzialità di sviluppo inesplorate oltre che per il carattere multi-provinciale e multi-regionale (Garda) e multi-locale (Ticino). Si tratta inoltre di due Sistemi turistici che hanno un peso decisamente rilevante dell'ambito della Macro Regione Europea dei laghi del Nord, sia in termini di strutture che di flussi turistici (si veda in proposito le Tabb. 1 e 2).

\subsubsection{L'ESTmL del Lago di Garda}

Il sistema gardesano raggruppa più di 40 comuni (25 rivieraschi) distribuiti in tre sponde, quella Bresciana, Veronese e Trentina. Tale sistema turistico che attrae ogni anno circa 3,8 milioni di turisti che si distribuiscono in 3.788 esercizi alberghieri ed extra alberghieri, per un totale di 19 milioni di presenze circa.

Tab.1: Capacità ricettiva alberghiera ed extralberghiera dei principali Laghi della Macro-Regione Europea dei Laghi del Nord Anno 2010 e variazione \% 2003/10

\begin{tabular}{|l|c|c|c|c|c|c|c|c|}
\hline & \multicolumn{4}{|c|}{ Alberghiero } & \multicolumn{4}{c|}{ Extra - Alberghiero } \\
\hline & Strutture & $\begin{array}{c}\Delta \\
\mathbf{0 3 / 1 0}\end{array}$ & Letti & $\begin{array}{c}\Delta \\
\mathbf{0 3 / 1 0}\end{array}$ & Strutture & $\begin{array}{c}\Delta \\
\mathbf{0 7 / 1 0}\end{array}$ & Letti & $\begin{array}{c}\Delta \\
\mathbf{0 7 / 1 0}\end{array}$ \\
\hline Garda & 1.007 & $0,30 \%$ & 65.817 & $20,93 \%$ & 2.781 & $9,53 \%$ & 126.602 & $7,91 \%$ \\
\hline Como & 190 & $1,60 \%$ & 10.160 & $11,93 \%$ & 1.196 & $51,78 \%$ & 15.588 & $-6,01 \%$ \\
\hline Maggiore & 214 & $-3,60 \%$ & 13.311 & $10,41 \%$ & 217 & $16,04 \%$ & 28.805 & $0,86 \%$ \\
\hline Ticino & 499 & $-11,01 \%$ & 20.410 & $-12,96 \%$ & N.D. & - & N.D. & - \\
\hline
\end{tabular}

Fonte: Elaborazione dell'Osservatorio laghi su dati Istat e O-Tur, 2010 http://users2.unimi.it/osservalaghi/ 
Tab. 2: Capacità ricettiva alberghiera ed extralberghiera dei principali Laghi della Macro-Regione Europea dei Laghi del Nord Anno 2010

\begin{tabular}{|l|c|c|c|c|}
\hline & \multicolumn{2}{|c|}{ Alberghiero } & \multicolumn{2}{c|}{ Extra - Alberghiero } \\
\hline & arrivi & presenze & arrivi & presenze \\
\hline Garda & 2.470 .931 & 9.104 .830 & 1.527 .808 & 10.053 .696 \\
\hline Como & 260.763 & 824.417 & 62.960 & 435.578 \\
\hline Maggiore & 523.880 & 1.492 .951 & 248.318 & 1.483 .337 \\
\hline Ticino & 1.106 .816 & 2.487 .164 & N.D. & 2.021 .372 \\
\hline
\end{tabular}

Fonte: Elaborazione dell'Osservatorio laghi su dati Istat e O-Tur, 2010 http://users2.unimi.it/osservalaghi/

Quanto alle performance del sistema turistico gardesano, basta qui ricordare come nel periodo 2003/2010 le presenze e gli arrivi alberghieri siano aumentate rispettivamente del $30,3 \%$ e del $23,2 \%$, quelle extralberghiere rispettivamente del $34,5 \%$ e del $24 \%$ circa. Nello stesso periodo il Tasso di occupazione Lordo alberghiero è passato dal $37 \%$ al $38 \%$ e quello extralberghiero dal $20 \%$ al $22 \%$, confermando la tenuta del sistema gardesano anche in periodi di crisi.

\subsubsection{L'ESTmL del Ticino}

Nel territorio del Canton Ticino vi sono due laghi: il Ceresio (Lago di Lugano) e la parte più settentrionale del lago Maggiore. Dal punto di vista istituzionale, il Canton Ticino è suddiviso in 11 Enti Turistici Locali a loro volta raggruppati in 4 regioni turistiche (Bellinzona e Alto Ticino, Lago di Lugano, Lago Maggiore e Valli e Mendrisiotto).

L'ESTmL dei laghi del Ticino si caratterizza per la presenza di 500 strutture alberghiere che nel 2010 hanno registrato un totale 2,5 milioni di presenze (si veda la Tab. 2) in trend di diminuzione (tra il 2000 e il 2010 gli arrivi sono diminuiti del $9.6 \%$ e le presenze del $18.8 \%$, con la conseguente contrazione dell'offerta del $16 \%$ circa (Tab.1).

\subsection{Metodologia della ricerca}

I dati sono stati raccolti attraverso un'indagine diretta (questionario CAWI) presso un campione di operatori alberghieri ed extralberghieri. L'analisi ha approfondito da un lato il comportamento della domanda (criteri di scelta, aspettative, comportamenti d'uso, motivi di soddisfazione/insoddisfazione); dall'altro i comportamenti degli operatori (caratteristiche strutturali, strategie, marketing e performance) e la rilevanza dei network relazionali tra i diversi stakeholder.

Il questionario proposto è stato costruito sulla base dei risultati di una prima indagine condotta nel 2009 e di numerosi incontri con i principali stakeholder del sistema turistico della Macro-Regione dei laghi del Nord-Italia (Associazioni degli albergatori, enti pubblici, ecc.) ed un workshop nel quale si sono incontrati le 
principali istituzioni, gli operatori turistici e alcuni rappresentanti del mondo accademico specializzati sul turismo. Tali attività hanno permesso di individuare il set di driver rilevanti per la competitività di tale sistema e la relativa valorizzazione da parte della domanda (47 variabili osservabili).

Il questionario è stato inviato a 1100 strutture ricettive ${ }^{5}$ di tutta l'area gardesana. Il tasso di risposta è stato del 14\%, pari a 156 strutture ricettive (gli esercizi alberghieri rappresentano il $41 \%$ del totale e gli extra-alberghieri il 59\%). Nel caso del Ticino, il tasso di risposta è stato dell'11\% per un totale di 113 rispondenti, di cui 46 hotel $(40.7 \%)$ e 67 strutture paralberghiere $(59.3 \%)$

\subsection{Strumenti analitici, formulazione del modello e risultati}

Come accennato, l'obiettivo di questo lavoro è quello di integrare gli attuali modelli di analisi della competitività delle destinazioni turistiche, in particolare esaminando le relazioni tra le sue diverse componenti. Ciò è stato effettuato attraverso lo studio di una dimensione "latente", che qui definiamo competitività turistica, in grado di sintetizzare i singoli fattori osservabili che caratterizzano un ecosistema turistico multi-locale, cercando di capire se questi sottendono uno o più costrutti teorici comuni non osservabili (le 7 macro variabili) e come questi si riflettono sul livello di competitività complessiva del sistema.

Per tal motivo si è reso necessario l'utilizzo di uno strumento in grado di poter fornire una struttura matematica più complessa al modello presentato, tale strumento è rappresentato dal Modello di Equazioni Strutturali (SEM) che permette di studiare simultaneamente la struttura delle interconnessioni esistenti tra variabili esplicative osservate (le 47 sottovariabili) e fattori latenti, sintesi di singoli fattori osservati (le 7 macro-variabili e la competitività). Tale strumento è peraltro già stato applicato in diversi studi del settore turistico ${ }^{6}$.

In particolare, in una prima fase il modello teorico ipotizzato è stato tradotto in un sistema di equazioni e relazioni tra variabili (Fig. 2).

Il nucleo del modello è formato dalla variabile latente $\left(\eta_{8}\right)$ Competitività che è collegata con un nesso causale diretto con le altre 7 variabili latenti $\left(\eta_{1} \ldots \eta_{7}\right)$, tali legami sono indicati dai coefficienti $\beta$.

5 Su un totale di 3918 strutture, tenendo in considerazione che di queste 2366 sono alloggi in affitto e quindi spesso più unità fanno capo ad una sola persona o agenzia e dunque non è stato possibile reperire tutti gli indirizzi mail. Inoltre vi è ancora una piccola percentuale di strutture che non possiedono il sito internet.

6 Ad esempio per testare la relazione tra le motivazioni di viaggio dei turisti e la destinazione scelta o più in generale le percezioni dei turisti (Rangaswamy et al., 2009; o gli effetti strutturali di quattro fattori di impatto turistico (perceived tourism development impacts, environmental attitudes, place attachment, development preferences about destination attractions/resources, and support for destination competitive strategies) sull'impatto turistico totale e sul supporto dei residenti allo sviluppo turistico (Yoon, et al., 2001). 
Fig. 2: Rappresentazione grafica del modello

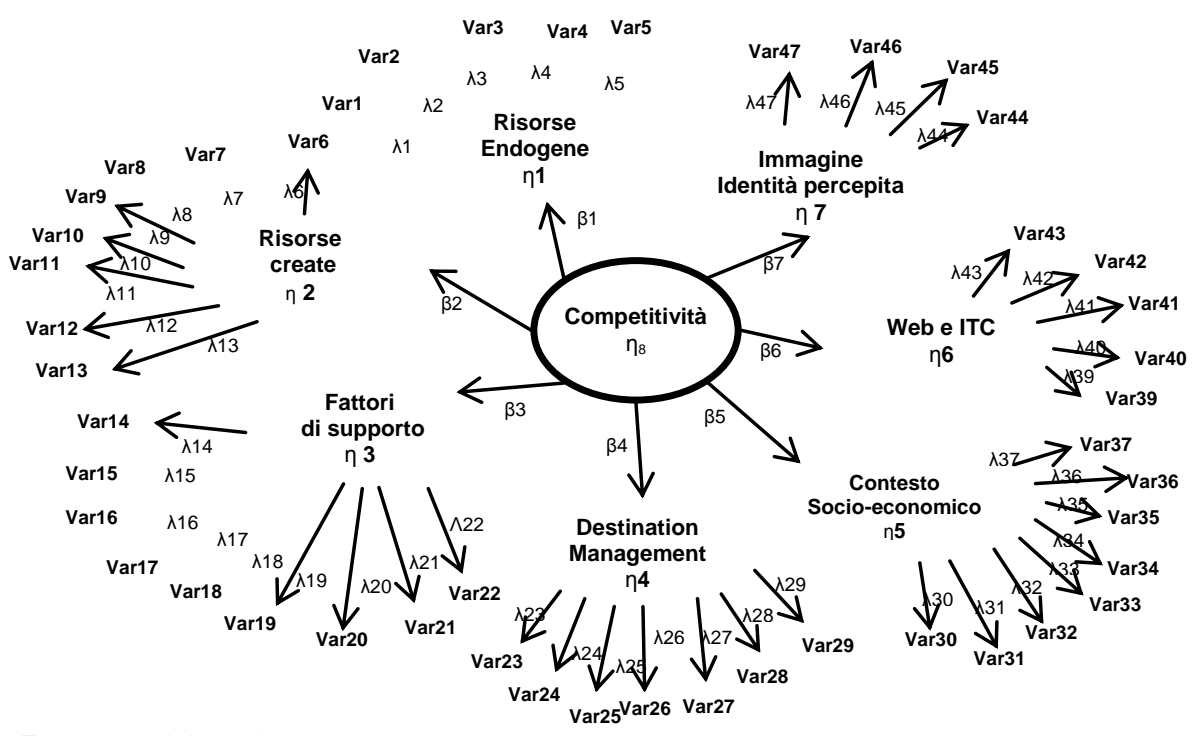

Fonte: ns. elaborazione

Le variabili latenti $\left(\eta_{1} \ldots \eta_{7}\right)$ derivano a loro volta dalle 47 variabili osservate. In particolare le variabili Var1, Var2, Var3, Var4, Var5, sono gli indicatori della variabile latente $\eta 1$; le variabili $\operatorname{Var} 6, \ldots .$. , Var13 della variabile latente $\eta 2$; le variabili Var14, ..., Var22 della variabile latente $\eta 3$; le variabili Var23, ..., Var29 della variabile latente $\eta 4$; le variabili $\operatorname{Var} 30, \ldots ., \operatorname{Var} 38$ della variabile latente $\eta 5$; le variabili Var39, ..., Var43 della variabile latente $\eta 6$; le variabili Var43, ..., Var47 della variabile latente $\eta 7$. I coefficienti che legato le variabili latenti a quelle osservate sono i $\lambda x$. Gli indici Alpha Cronbach e $\rho$ Dillon-Goldstein hanno un buon livello di accettabilità $(>0,8)$, rilevando dunque un alto livello di correlazione tra le variabili e un elevato grado di consistenza interna in ogni blocco indice che il fattore latente riesce a spiegare bene le variabili osservate sottostanti. Inoltre gli indici GFIgarda (0,875), GFI-ticino (0,775), AGFI-garda (0,8243), AGFI-ticino (0,7456) indicano un buon adattamento del modello ai dati.

Successivamente è stata effettuata l'analisi dei coefficienti strutturali che ha permesso di valutare se il modello proposto è in grado di sostenere le ipotesi teoriche che lo costituiscono. La prima evidenza emergente è che il modello convalida le ipotesi teoriche in quanto presenta tutti coefficienti di correlazione positivi tra le variabili prese in esame, anche nel caso della variabile Web e ICT qui introdotta. Quanto ai coefficienti strutturali che legano le variabili osservate alle relative 7 macro-variabili e quelli che legano quest'ultime alla variabile "Competitività" dei due sistemi turistici considerati, questi sono evidenziati dal Fig. 3, mentre la Tab. 3 evidenzia l'impatto di ogni macrovariabile sulla "competitività" (coefficienti $\beta$ ). 
Fig. 3: Rappresentazione grafica del modello con i parametri stimati: STML Garda e Laghi Ticino

a) il caso dell'ESTmL del Garda

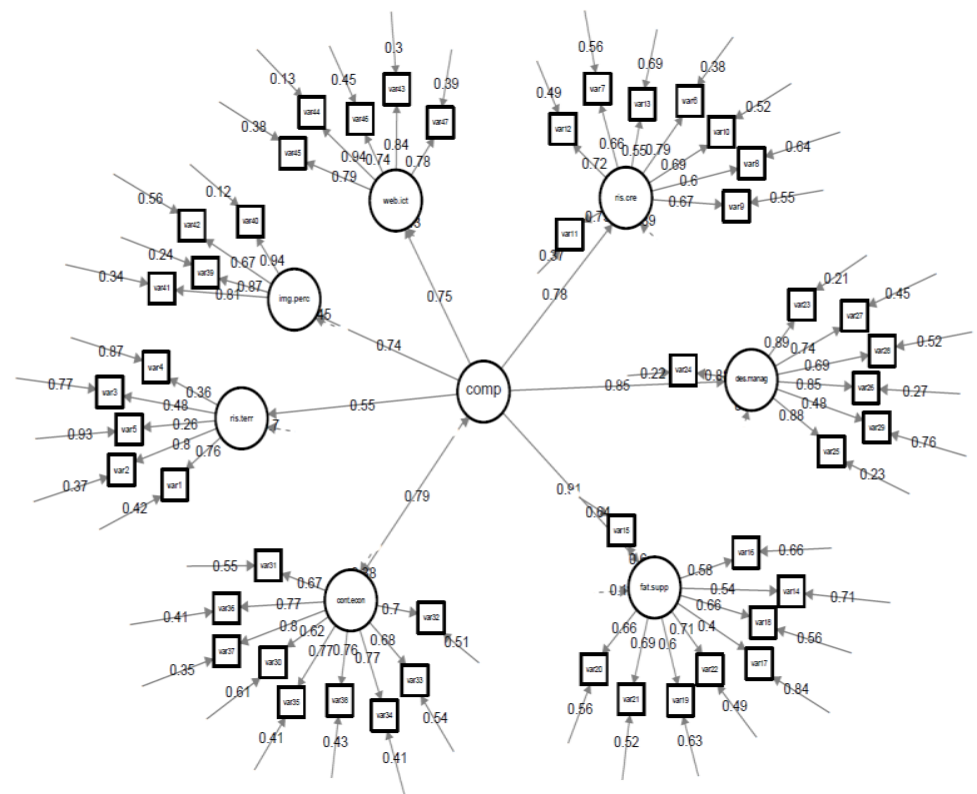

b) il caso dell'ESTmL del Ticino

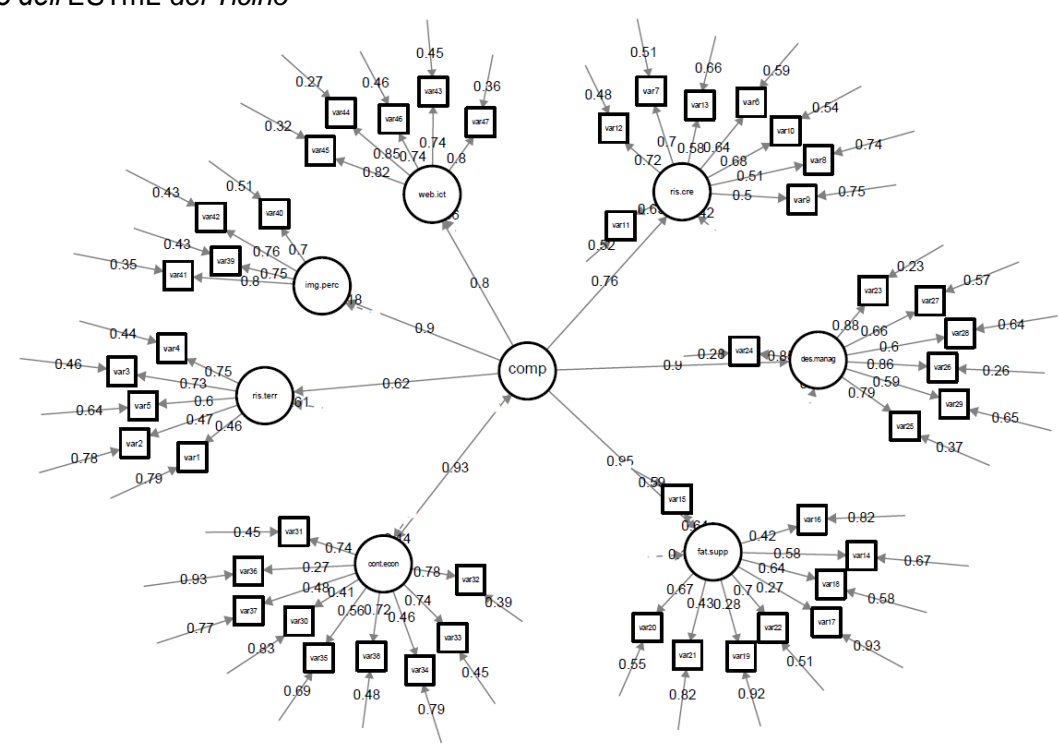

Fonte: ns. elaborazione 
Tab. 3: Livello di impatto delle macro-variabili sulla variabile "Competitività" dei Sistemi territoriali Garda e Ticino

\begin{tabular}{|l|c|c|}
\hline & GARDA & TICINO \\
\hline Risorse endowed & 0,55 & 0,62 \\
\hline Risorse created & 0,78 & 0,76 \\
\hline Fattori di supporto & 0,91 & 0,95 \\
\hline Destination management & 0,85 & 0,9 \\
\hline Contesto economico & 0,79 & 0,93 \\
\hline Immagine percepita & 0,74 & 0,9 \\
\hline Web e ICT & 0,75 & 0,8 \\
\hline
\end{tabular}

Fonte: ns. elaborazione

In particolare, come si osserva dalla tabella 3, nel caso del Garda emerge chiaramente che la Competitività è fortemente influenzata dai Fattori di supporto e dal Destination Management vale a dire dallo sviluppo di fattori che facilitano la fruizione dei servizi turistici e di adeguate politiche d'indirizzo e coordinamento.

Le variabili contesto socio-economico, risorse create, Web-ICT e immagine e identità percepita hanno un medio impatto sulla variabile competitività. Questi risultati confermano le aspettative: il contesto socio-economico (sicurezza dei luoghi, stabilità economica e politica) è un attrattore essenziale della domanda e influenza le scelte d'investimento delle imprese; le risorse create sono rilevanti nella realizzazione dell'intera esperienza turistica; il Web e l'ICT rispondono alle nuove logiche comportamentali dei turisti (internet quale fonte primaria di informazione e connessione); l'immagine e l'identità percepita influenzano le scelte della domanda, scelte che come si è detto sono sempre più condivise e "virali". Per contro, risulta piuttosto inaspettato il ridotto impatto delle risorse endowed. Tali risorse insieme ai fattori d'offerta turistica vengono generalmente considerati importanti forze generatrici di domanda (Formica e Uysal, 1998) e soprattutto primarie determinanti dall'attrattività turistica (Crouch, 2006). Da qui la considerazione che anche i sistemi turistici non dotati di risorse naturali attrattive, di clima favorevole, di risorse culturali e tradizioni forti (variabili non o difficilmente acquisibili e/o migliorabili), possono incrementare la propria competitività agendo sugli altri driver.

\section{Conclusioni}

Nel complesso tale modello risulta efficace nel determinare sia i driver della competitività degli ecosistemi multilocali, sia le policy territoriali e multi territoriali necessarie per migliorarne la competitività e ridurne le eventuali asimmetrie emergenti. Per una sua effettiva validazione è tuttavia necessaria la sua applicazione a differenti contesti. In tale direzione, come si è accennato, è stata effettuata una prima convalida applicandolo all'ESTlm del Ticino. Anche in questo caso la SEM ha confermato che $\mathrm{i}$ fattori che maggiormente incidono sulla competitività 
complessiva sono i fattori di supporto e il Destination Management (si veda la Tab.3) rilevando dunque, come per il sistema Gardesano, che un sistema competitivo debba saper offrire efficienti facilitazioni che permettano ai turisti di poter usufruire dei servizi turistici garantendo, ad esempio, la possibilità di facili spostamenti sia con mezzi pubblici che privati, una location pulita ed ordinata, strutture sanitarie adeguate, centri di informazione e assistenza efficienti. Ma tutto ciò è condizionato da un'efficiente attività di indirizzo e coordinamento dei Destination Manager. Si può allora evincere che un intervento primario su tali due fattori sia essenziale al fine di incrementare la competitività del sistema. Tuttavia nel caso Ticinese emerge che le variabili contesto socio economico e l'immagine e l'identità percepita hanno un peso decisamente superiore rispetto a quanto emerso per il Garda, dovuto principalmente al migliore background normativo e relazionale che disciplina il contesto Svizzero. È inoltre confermata la relativa minor rilevanza delle risorse endowed e di quelle created, confermando dunque l'ipotesi secondo la quale le risorse naturali e territoriali non sono più sufficienti per affrontare in modo vincente la crescente competitività turistica, ma si rende necessaria una governance efficace che crei soddisfazione e fiducia tra i diversi stakeholder.

Va peraltro segnalato che il modello si basa su valutazioni degli stakeholder e dunque potrebbe essere "viziato" da fattori soggettivi e tuttavia "compensato o attenuato" nei grandi numeri. Tale limite potrebbe essere superato individuando per i diversi driver indicatori oggettivi. Sarebbe inoltre opportuno ripetere la ricerca a "rolling", al fine di testare la validità del modello nel tempo anche al variare di condizioni esterne e per evidenziare l'evoluzione dei fattori e della loro influenza sulla competitività.

\section{Bibliografia}

AMBLER T. (1995), "Brand equity as a relational concept", The Journal of Brand Management, vol. 2, n. 6, pp. 387.

BARNEY J. (1991), "Firm resources and sustained competitive advantage", Journal of management, Sage Publications, vol. 17, n. 1, 99-120.

BUHALIS B., (2000), "Marketing the competitive destination of the future", Tourism Management, n. 21, pp. 97-116.

CAROLI M.. (1999), Il Marketing territoriale, Franco Angeli, Milano.

CAROLI M. (2009), "Un modello di valutazione della qualità di una destinazione turistica", Economia dei Servizi, vol. 4, n. 1, pp. 29-46.

CROUCH G.I., RITCHIE J.R.B. (1999), "Tourism, Competitiveness, and societal prosperity", Journal of Business Research, n. 44, vol. 3, pp. 137-152.

CROUCH G.I., RITCHIE J.R.B. (2003), "The competitive destination: a sustainable tourism perspective", Wallingford, CABI.

CROUCH G.I. (2006), "Destination competitiveness: insight in to attribute importance", International conference on trends, impacts and policies on tourism development, Crete.

DWYER L., KIM C. (2003) "Destination competitiveness: A model and Determinants", Current Issues in Tourism, vol. 6, Issue 5 October, pp. 369-414. 
ENRIGHT M.J., NEWTON J. (2005), "Determinants of tourism destination competitiveness in Asia Pacific: comprehensiveness and universality", Journal of Travel Research, vol. 43, n. 4, pp. 339-350.

FLAGESTAD A., HOPE C.A. (2001), "Strategic success in winter sports destinations: a sustainable value creation perspective", Tourism management, vol. 22, n. 5 .

FLORIDA R. (2009), Who's Your City?: How the Creative Economy Is Making Where to Live the Most Important Decision of Your Life, Vintage Canada.

FORMICA S., UYSAL M. (1998). "Market segmentation of an international culturalhistorical event in Italy", Journal of Travel Research, vol. 36, n. 4, pp. 16-24.

FOURNIER S. (1998), "Consumer and their Brands: Developing Relationship Theory in Consumer Research", Journal of Consumer Research, vol. 24, n. 3, pp. 343-373.

GOVERS R., GO F.M. (1999), “ Achieving Service Quality Through the Application of Importance-Performance Analysis”, Service Quality and Management, pp. 161-185.

GRÖNROOS C. (1994), Marketing e management dei servizi, Isedi, Torino.

KOTLER P., GERTNER D. (2002), "Country as Brands, Product, and Beyond: A Place Marketing and Brand Management Perspective", Brand Management, vol. 9, n. 4-5, pp. 249-261.

MURPHY P., PRITCHARD M.P., SMOTH B. (2000), "The destination product and its impact on traveller perceptions", Tourism Management, vol. 21, n. 1, pp. 43-52.

OSSERVATORIO LAGHI SU DATI ISTAT E O-TUR (2010), http://users2.unimi.it/osservalaghi/

OSTILLIO M.C. (2000), "La comunicazione territoriale", in Valdani E., Ancarani F., (a cura di), Il marketing territoriale, logiche, strumenti e casi nel contesto italiano e internazionale, Egea, Milano.

PILOTTI L. (2001), "Valore di sistema e rigidità flessibili. La città-territorio verso la virtualità. Competenze, reti multilivello e communities per un valore di sistema tra identità e trasferibilità", Sviluppo Locale, vol. VIII, n. 18, pp. 3-40.

PILOTTI L. (2006), Eterogeneità delle imprese e varietà dei modelli organizzativi, Franco Angeli, Milano.

PILOTTI L., GANZAROLI A. (2009), Proprietà condivisa e open source : il ruolo della conoscenza in emergenti ecologie del valore, Franco Angeli, Milano.

PILOTTI L., TEDESCHI-TOSCHI A., APA R. (2011), "La competitività ecologica dei sistemi territoriali tra identità, immagine e vantaggi "costruiti". Quale marketing dei servizi turistici di fronte alla "long tail"?", in The proceedings of 10th International Conference Marketing Trends, Paris-Venice, Marketing Trends Association.

PILOTTI L., TEDESCHI-TOSCHI A., APA R.(2010), "Which quality for the system competitiveness? The "Long-Tail" touristic service case", International Conference Quality and Service Sciences. $13^{\text {th }}$ Toulon-Verona Conference. Coimbra 2-4 Settembre.

POON A. (1993), "Tourism, Technology, and Competitive Strategies", Cab International, Wallingford.

PRAHALAD C.K., HAMEL G. (1990), "The Core Competence of the Corporation”, Harvard Business Review, May-June, pp. 79-91.

RISPOLI M., TAMMA M. (1995), "Risposte strategiche alla complessità: le forme di offerta dei prodotti alberghieri”, Giappichelli, Torino.

RANGASWAMY N., CHAIBOONSRI C., CHAITIP P. (2008), "A Structural Equation Model: India's International Tourism Demand for Tourist Destination", Annals of the University of Petrosani, Economics, vol. 8, n. 2, pp. 107-134. 
SENN L. (2002), "New economy, territorio e fiscalità", Scienze Regionali, vol. 1, n. 1, pp. 147-156.

VALDANI E., ANCARANI F.(2000), Il marketing territoriale, logiche, strumenti e casi nel contesto italiano e internazionale, Egea, Milano.

VALDANI E., GUENZI P. (1998), "Il marketing nei parchi tematici", Egea, Milano.

VAN DEN BERG L., BRAUN E. (1999), "Urban competitiveness, marketing and the need for organizing capacity”, Urban Studies, vol. 36, pp. 987-999.

WEAVER D., OPPERMANN M., (2000), Tourism Management, John Wiley and Sons, Brisbane.

YOON Y., GURSOY D., CHEN J.S. (2001), "Validating a tourism development theory with structural equation modeling", Tourism Management, Elsevier, vol. 22, n. 4, pp. 363 372 . 